\title{
Focus in Honor of Alexander Makarov, Recipient of the 2008 Award for a Distinguished Contribution in Mass Spectrometry
}

$\mathrm{T}$ The 2008 Award for a Distinguished Contribution in Mass Spectrometry was awarded to Dr. Alexander Makarov at the $56^{\text {th }}$ ASMS Conference for his discovery and development of the Orbitrap ${ }^{\mathrm{TM}}$ mass analyzer. The Orbitrap mass analyzer affords outstanding performance with respect to mass accuracy, resolving power, sensitivity, and dynamic range. The commercialization of this technology by Thermo Fisher Scientific resulted in tremendous impact and acclaim for this new type of mass spectrometer and sweeping recognition for the significance of Alexander's discovery.

Soon after he was born in Irkutsk, a town in Siberia, in 1966, Alexander exhibited a keen interest in technology and applied science. He pursued his undergraduate education at the Moscow Engineering Physics Institute, followed by masters and doctoral degrees (in 1992) at the same institution where he focused on the design of time-of-flight mass spectrometers. Thereafter, he joined the General Physics Institute of the Russian Academy of Sciences and also became a consultant on ion optics to Kratos Analytical. He undertook a post-doctoral position at Warwick University before uniting with several former employees of Kratos at HD Technologies, a small high-tech company in Manchester where Alexander first initiated his work on the Orbitrap. Despite several funding difficulties, HD Technologies won a small SMART award from the UK government via an application submitted by Steve Davis, and this allowed Alexander to construct the first Orbitrap with assistance from technical experts R. Lawther, A. Hoffman, and S. Davis in 1996 to 1997.

The original roots of the Orbitrap stem from the principle of orbital trapping, the ability to trap charged particles in electrostatic fields, defined by Kingdon in 1923. Alexander used the term "Orbitrap" to describe a type of mass analyzer in which ions rotate around an electrode while undergoing harmonic oscillations along the axis of rotation at frequencies related to their $\mathrm{m} / \mathrm{z}$ values. Fourier transformation of the time domain signal produced from detection of the image current of the coherent oscillating ions yields the mass spectrum.

The first spectra were obtained in 1998, and a resolving power of 160,000 was achieved in 1999. Thermo Fisher Scientific purchased HD Technologies in 2000, an event

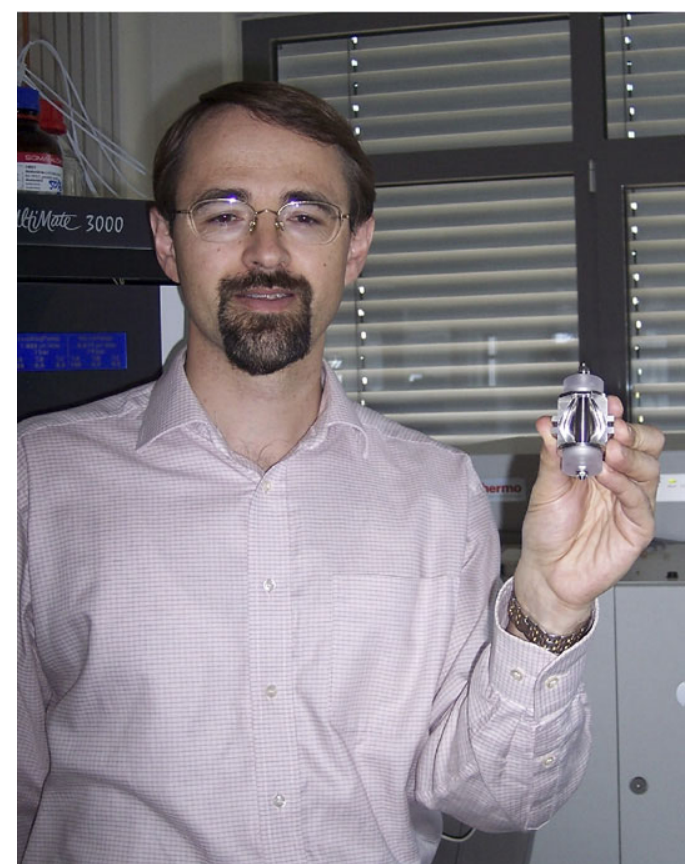

that allowed Alexander to continue to develop the Orbitrap, in conjunction with expansion of the Orbitrap research team, including M. Hardman, A. Kholomeev, and E. Denisov. Numerous challenging technical problems were overcome even as the project team was transferred to Bremen in 2002. A prototype Orbitrap was installed in Professor Graham Cooks' laboratory in early 2003. Development of the Orbitrap accelerated with the additional expertise offered by S. Horning, W. Balschun, O. Lange, F. Czemper, G. Jung, and others. The outstanding efforts of the team, largely led by Alexander Makarov, culminated in the commercial release of the LTQ Orbitrap tandem mass spectrometer at the ASMS Conference in San Antonio, TX, in 2005. The Orbitrap enjoyed enormous commercial success immediately, and it is a tribute to Alexander Makarov's persistence and ingenuity that the entire inception, development and commercialization occurred within an amazingly short period including only six years of experimentation. 
Alexander's colleagues and friends in the mass spectrometry community express their highest regards for Alexander's achievements and their respect for his passion for science. Dr. Stevan Horning, Director of Global R\&D LSMS, Life Science Mass Spectrometry at Thermo Fisher Scientific, in Bremen, Germany, notes that his first interaction with Alexander stemmed from a simple request from him for a particular part, a pre-amplifier from an Odyssey FTMS instrument. Stevan's group, which was developing its own FTbased technology, the eventual LTQ FT mass spectrometer, at that same time, responded by sending him an entire Odyssey data system. Owing to the relative proximity of Bremen to Manchester, Stevan's group visited Alexander at HD Technologies shortly thereafter. Stevan recalls the first meeting as extremely memorable with Alexander exhibiting great generosity in sharing the development of the Orbitrap and his vision for the potential impact of this technology. After that first meeting, a working relationship was established based on the conviction that the Orbitrap had the potential to be an enormous success that aligned with the infrastructure already established for the LTQ FT instrument. When HD Technologies was bought by Thermo Fisher Scientific, the Orbitrap technology was transferred to Bremen, as Stevan notes, and a large team of engineers moved forward with the mechanical and electrical tasks of taking "one-off" designs and developing them into parts that could be used in series production. During the developmental phase of the Orbitrap in Bremen, Alexander commuted frequently between Bremen and Manchester. In Manchester, Alexander undertook calculations on Orbitraps and examined experimental data. When in Bremen, he worked relentlessly with E. Denisov on the instrument, characterizing and modifying parts. Stevan recalls that commuting was a hardship for Alexander and his family, but a sacrifice that Alexander was willing to make in the short term to accelerate the development of the Orbitrap. In Stevan's words, "Alexander is very intense in his research and work, but he places equally high value on spending time with his wife and two children. Not often does one encounter a genius, and even less often does one have the opportunity to work with one. It is a real pleasure for scientists and engineers at Thermo Fisher Scientific to work with Alexander. I know his friends in the mass spectrometry community feel the same, and as a community we are very appreciative of his Orbitrap invention. It has made mass spectrometry possibly the strongest analytical tool for studies in proteomics and biology. The best news though, is we can look forward to even more from Alexander." Steve Davis, currently Managing Director at PD Analytical Pty. Ltd., echoes these sentiments and recollects that "the Orbitrap prototype was such an intriguing blue sky concept ... if anyone could make it work Alexander could." Steve also recalls that some of the initial visitors from Thermo Fisher Scientific had confessed skepticism upon reading the original Orbitrap patent, even sug- gesting "There is no way that is going to work" but exhibiting respectful scientific enthusiasm upon seeing the spectra that emerged from the prototype Orbitrap. Throughout the long hours and stressful times during which the Orbitrap was developed, Alexander's colleagues enjoyed his remarkable disposition. After addressing unfortunate results with his trademark phrase "Well ..., this is disappointing", made all the more memorable with his Russian accent, Alexander would inevitably reveal success and renewed inspiration with the long awaited "Ohhhhhh ... beauty".

This Special Focus Issue on "The Orbitrap" is dedicated to Alexander Makarov and contains eleven original articles. This issue opens with an article, authored by Alexander Makarov, that assesses the performance of a high-field Orbitrap in which a decrease in the gap between the inner and outer Orbitrap electrodes affords higher field strengths that allow a resolving power over 350,000 for ions of $\mathrm{m} / \mathrm{z} 524$ and isotopic resolution of proteins over $40 \mathrm{kDa}$. The second article, authored by Robert Noll et al., describes the use of resonant excitation for improved control of ion packets inside the Orbitrap based on rephasing the ion population, ultimately leading to better resolution and peak shape. Catherine Wong et al. evaluate the impact of signal threshold parameters of the Orbitrap associated with data dependent spectra sampling for identification of peptides and proteins and report that the higher mass accuracy of the Orbitrap improves database searching and peptide validation software performance. In the fourth article, a top-down approach for the accurate mass determination and partial amino acid sequencing of monoclonal antibody protein pharmaceuticals is described by Pavel Bondarenko et al. In the next article, Jarrod Marto et al. assess the quantification of iTRAQlabelled phosphopeptides by using higher energy dissociation (HCD) in an Orbitrap mass spectrometer. In the sixth article, Josh Coon et al. report a post-acquisition processing method for ETD-based spectra that improves the confidence and number of peptide identifications based on removal of interfering peaks prior to database searches. A benchtop Orbitrap mass spectrometer is used to evaluate qualitative and quantitative data from microsomal stability and in vivo pharmacokinetics studies by Kevin Bateman et al. in the following article. Kerstin Strupat et al. assess the results of coupling a MALDI source to an Orbitrap mass spectrometer in conjunction with a collisional cooling interface in the eighth article. An Orbitrap mass spectrometer is used for quantitative and qualitative residue analysis in food and feed matrices by Markus Kellman et al. In the tenth article, Matthias Mann et al. describe a new approach for the determination of mass accuracy for protein identification by using an Orbitrap. The final article by Alexander Makarov closes the special focus. In that article, the performance of an Orbitrap for analysis of large, intact protein ions is examined. It is determined that both the ion loss due to dissociation and scattering-induced de-phasing of ion packets con- 
tributes to a reduction in resolving power for higher mass ions.

The Orbitrap has gained universal acclaim as a landmark discovery and has had a substantial impact on the field of mass spectrometry. The Journal of the American Society for Mass Spectrometry is honored to present this focus section to highlight Alexander's contribution and the significance of the Orbitrap.

Jennifer S. Brodbelt Department of Chemistry and Biochemistry University of Texas Austin, Texas USA 